\title{
Implications of current constraints on parton charge symmetry
}

\author{
J T Londergan ${ }^{1}$ and A W Thomas $^{2}$ \\ ${ }^{1}$ Department of Physics and Nuclear Theory Center, Indiana University, Bloomington, \\ IN 47405, USA \\ 2 Jefferson Laboratory, 12000 Jefferson Ave., Newport News, VA 23606, USA \\ E-mail: tlonderg@indiana.edu and awthomas@jlab.org
}

Received 1 July 2005

Published 24 August 2005

Online at stacks.iop.org/JPhysG/31/1151

\begin{abstract}
For the first time, charge symmetry breaking terms in parton distribution functions have been included in a global fit to high energy data. We review the results obtained for both valence and sea quark charge symmetry violation and compare these results with the most stringent experimental upper limits on charge symmetry violation for parton distribution functions, as well as with theoretical estimates of charge symmetry violation. The limits allowed in the global fit would tolerate a rather large violation of charge symmetry. We discuss the implications of this for various observables, including extraction of the Weinberg angle in neutrino DIS and the Gottfried and Adler sum rules.
\end{abstract}

\section{Introduction}

Charge symmetry is a restricted form of isospin invariance involving a rotation of $180^{\circ}$ about the ' 2 ' axis in isospin space. For parton distributions, charge symmetry involves interchanging up and down quarks while simultaneously interchanging protons and neutrons. In nuclear physics, charge symmetry is generally obeyed at the level of a fraction of a percent $[1,2]$. Charge symmetry violation in parton distribution functions (PDFs) arises from two sources; from the difference $\delta m \equiv m_{d}-m_{u}$ between down and up current quark masses, and from electromagnetic (EM) effects.

Since charge symmetry is so well satisfied at lower energies, it is natural to assume that it holds for parton distributions. Introducing charge symmetry reduces by a factor of two the number of PDFs necessary to describe high-energy data. There is no direct experimental evidence for violation of charge symmetry in parton distributions. For these reasons, until recently all phenomenological PDFs have assumed charge symmetry at the outset. In section 2, we review the experimental evidence that places the strongest upper limits on charge symmetry violation (CSV) in PDFs. In section 3, we review the recent global fit by Martin et al [3] that includes for the first time the possibility of charge symmetry violating PDFs for both valence 
and sea quarks. We compare the results of this phenomenological fit with the experimental limits on parton CSV as well as with theoretical estimates of both the magnitude and sign of charge symmetry violating parton distributions.

In section 4, we review the effect of isospin violating PDFs on various observables. We first review the extraction of the Weinberg angle by the $\mathrm{NuTeV}$ collaboration [4]. This observable turns out to be quite sensitive to valence quark CSV contributions. In fact, effects allowed by the phenomenological MRST CSV PDFs are sufficiently large that they could account for the entire NuTeV anomaly. In section 5 we discuss the implications of CSV in PDFs for various partonic sum rules. Here, there could be important contributions from sea quark CSV; this is discussed with respect to the Gottfried and Adler sum rules. In the final section we present our conclusions.

\section{Experimental limits on parton charge symmetry violation}

The most stringent test of parton charge symmetry comes from comparing the structure function $F_{2}^{\gamma}$ for charged lepton DIS with the structure function $F_{2}^{W N_{0}}$ from neutrino chargedcurrent reactions, measured on isoscalar targets $N_{0}$. In leading order, assuming parton charge symmetry, the structure functions have the form [5]

$$
\begin{aligned}
& F_{2}^{\gamma N_{0}}(x) \approx \frac{5 Q(x)}{18}+\frac{x}{6}(c(x)+\bar{c}(x)-s(x)-\bar{s}(x)) \\
& F_{2}^{W^{ \pm} N_{0}}(x) \approx Q(x) \pm x(s(x)-\bar{s}(x)+\bar{c}(x)-c(x)) \\
& Q(x)=\sum_{j=u, d, s, c} x\left(q_{j}(x)+\bar{q}_{j}(x)\right) .
\end{aligned}
$$

In equation (1), $F_{2}^{W^{ \pm} N_{0}}$ is the structure function for charged-current processes induced by $v(\bar{v})$ on an isoscalar target. Charge symmetry violation and NLO effects are not included in this equation. In the limit of exact charge symmetry, there is a simple relation between the charged-lepton and neutrino structure functions, corrected for heavy quark effects. This relation is defined as the 'charge ratio' $R_{c}\left(x, Q^{2}\right)$ or the ' $5 / 18$ rule.' Expanding $R_{c}$ to lowest order in the charge symmetry violating terms gives

$$
\begin{aligned}
R_{c}(x) & \equiv \frac{F_{2}^{\gamma N_{0}}(x)+x(s(x)+\bar{s}(x)-c(x)-\bar{c}(x)) / 6}{5 \bar{F}_{2}^{W N_{0}}(x) / 18} \\
& \approx 1+\frac{3 x(\delta u(x)+\delta \bar{u}(x)-\delta d(x)-\delta \bar{d}(x))}{10 Q(x)} .
\end{aligned}
$$

Equation (2) introduces the CSV parton distributions,

$$
\delta u(x)=u^{p}(x)-d^{n}(x), \quad \delta d(x)=d^{p}(x)-u^{n}(x),
$$

with analogous relations for antiquarks. In equation (2), $\bar{F}_{2}^{W N_{0}}$ is the average of $v$ and $\bar{v}$ charge-changing $F_{2}$ structure functions; it also requires strange and charm PDFs. Deviation of $R_{c}(x)$ from unity would be an indication of a non-zero charge symmetry violating contribution.

The most precise results for the charge ratio compare the CCFR $v-F e$ structure functions [6] with the NMC $\mu-D$ measurements [7, 8]. An initial analysis by the CCFR group [6] gave a value of $R_{c}(x)$ consistent with unity in the region $0.1 \leqslant x \leqslant 0.4$ at the $2-3 \%$ level. From equation (2), this sets an upper limit to parton CSV effects at about the 6-9\% level. However, for $x<0.1$ comparison of the $v$ and $\mu$ structure functions appeared to show a discrepancy that grew monotonically with decreasing $x$. The CCFR group re-analysed the neutrino data, and in the new analysis the small- $x$ discrepancy disappeared [9], largely as a result of two 
factors. The first was the treatment of charm mass corrections. In the initial analysis, these were incorporated using the 'slow rescaling' hypothesis due to Georgi and Politzer [10]. The re-analysis involved NLO calculations, which differed significantly from the slow-rescaling procedure at small $x$-as had been suggested by Boros et al [11].

The second significant effect involved separation of the $F_{2}$ and $F_{3}$ structure functions in charged-current $v$ DIS. The sum of $v$ and $\bar{v}$ charged-current DIS cross sections contains a linear combination of neutrino $F_{2}$ and $F_{3}$ structure functions:

$\frac{\mathrm{d}^{2} \sigma_{C C}^{\nu}}{\mathrm{d} x \mathrm{~d} y}+\frac{\mathrm{d}^{2} \sigma_{C C}^{\bar{v}}}{\mathrm{~d} x \mathrm{~d} y} \sim 2\left(1-y-y^{2} / 2\right) \bar{F}_{2}^{W N_{0}}\left(x, Q^{2}\right)+\left(y-y^{2} / 2\right) \Delta x F_{3}\left(x, Q^{2}\right)$.

In equation (4), in LO (assuming charge symmetry), $\Delta x F_{3} \approx 2 x(s+\bar{s}-c-\bar{c})$. In equation (4) we have dropped terms of order $m_{N}^{2} / s$, and for simplicity the L/T ratio, $R$, has been set to zero. In the initial analysis, data for a given $x$ bin were averaged over all $y$, and the $\Delta x F_{3}$ structure function was estimated using phenomenological PDFs. In the re-analysis both $\bar{F}_{2}$ and $\Delta x F_{3}$ were extracted [9]. The experimental values for $\Delta x F_{3}$ differed substantially from phenomenological predictions. This affected the extracted values for the $F_{2}$ neutrino structure functions. The combined effect of the NLO treatment of charm production, and the model-independent extraction of $\Delta x F_{3}$ removed the small- $x$ discrepancy. The charge ratio $R_{c}$ of equation (2) is now unity to within experimental error, even at small $x$.

\section{Phenomenological charge symmetry violating PDFs}

Because CSV effects are typically very small at nuclear physics energy scales $[1,2]$, theoretical expectations were that parton CSV would be below the $1 \%$ level [5]. This plus the lack of direct evidence for violation of parton charge symmetry meant that all previous phenomenological PDFs have assumed the validity of parton charge symmetry at the outset. However, Martin, Roberts, Stirling and Thorne (MRST) [3] have recently studied the uncertainties in parton distributions arising from a number of factors, including isospin violation.

The MRST group chose a specific model for valence quark charge symmetry violating PDFs. They chose a function of the form

$$
\delta u_{\mathrm{v}}(x)=-\delta d_{\mathrm{v}}(x)=\kappa f(x) \quad f(x)=(1-x)^{4} x^{-0.5}(x-0.0909) .
$$

The function $f(x)$ was chosen so that at both small and large $x, f(x)$ had a similar form to the MRST valence quark distributions. The first moment of $f(x)$ was fixed to be zero, in agreement with the valence quark normalization constraint

$$
\int_{0}^{1} \mathrm{~d} x \delta \mathrm{d}_{\mathrm{v}}(x)=\int_{0}^{1} \mathrm{~d} x \delta u_{\mathrm{v}}(x)=0 .
$$

Violation of equation (6) would be equivalent to changing the total number of valence quarks in the proton and/or neutron, which is unacceptable. The valence quark normalization condition requires that the CSV function has at least one node; MRST chose a simple function that agrees with the valence PDFs at both small and large $x$ and has zero first moment.

Inclusion of a valence quark CSV term changes the up and down quark distributions in the neutron with respect to their charge symmetric counterparts in the proton. This changes the momentum carried by valence quarks in the neutron from those in the proton, since the total momentum carried by valence quarks is given by the second moment of the distribution. For example, the momentum carried by up valence quarks in the neutron

$$
U_{\mathrm{v}}^{n} \equiv \int_{0}^{1} x u_{\mathrm{v}}^{n}(x) \mathrm{d} x .
$$



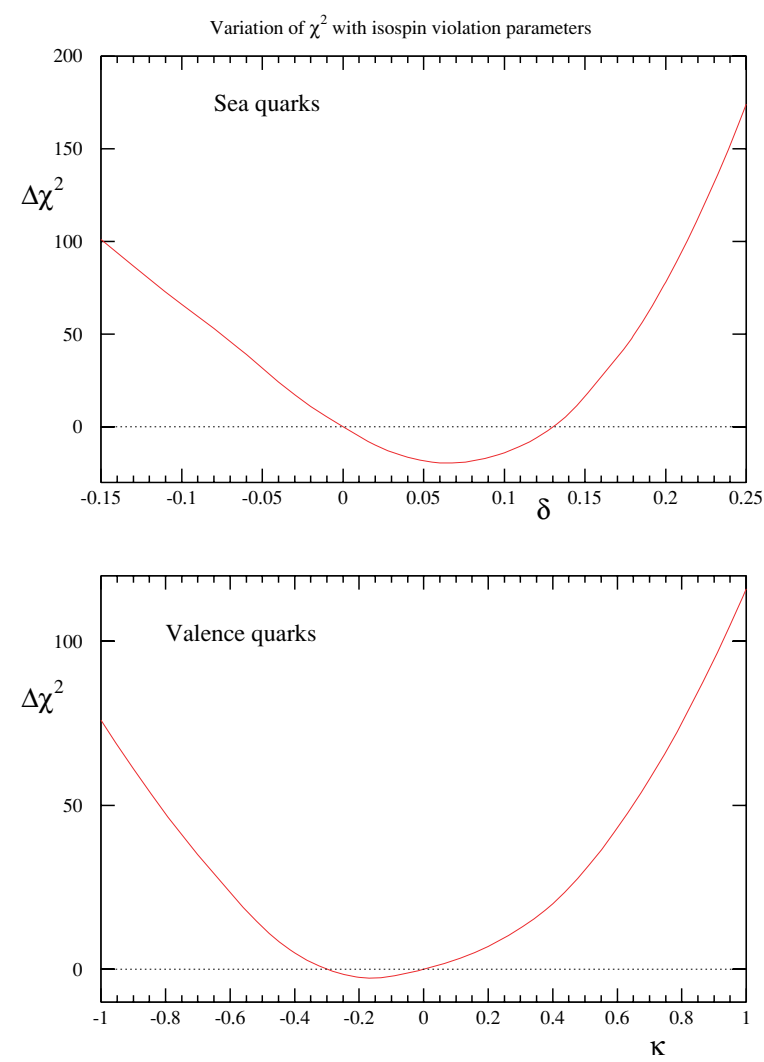

Figure 1. $\chi^{2}$ profile for phenomenological isospin violating parton distributions, for sea quarks (top curve) and valence quarks (bottom curve), from the MRST group [3]. The quantity $\delta$ associated with sea quark isospin violation is defined in equation (9) while the coefficient $\kappa$ is defined in equation (5).

Because the total momentum carried by valence (up plus down) quarks in the neutron, $U_{\mathrm{v}}^{n}+D_{\mathrm{v}}^{n}$, is determined to within about $2 \%$, MRST chose a functional form that kept this quantity relatively constant. For this reason, they insisted that the valence CSV terms for up and down quarks in the nucleon be equal and opposite. Note that QCD evolution does not preserve this relation, but it is very nearly maintained for the region of evolution of interest. The overall coefficient, $\kappa$, was then varied in a global fit to a wide range of high energy data. For simplicity, the CSV term was assumed to be the same for all $Q^{2}$ in the MRST global fit.

The value of $\kappa$ which minimized $\chi^{2}$ was $\kappa=-0.2$. The MRST $\chi^{2}$ versus $\kappa$ is shown as the bottom curve in figure 1 . Clearly $\chi^{2}$ has a shallow minimum with the $90 \%$ confidence level obtained for the range $-0.8 \leqslant \kappa \leqslant+0.65$. Since the form they chose for valence quark $\mathrm{CSV}$ is strongly constrained and the resulting $\chi^{2}$ minimum is quite shallow, one should not assign too much significance to the result. In global fits of this type, one should remember that, unless the shape of the constrained function is in close agreement with the actual parton distribution, the overall magnitude obtained in a global fit can be misleading.

However, the global fit MRST results can be compared with theoretical estimates of valence quark CSV. We know that charge symmetry violation will arise from the down-up quark mass difference, $\delta m$, plus some electromagnetic (EM) corrections. One feature of the 


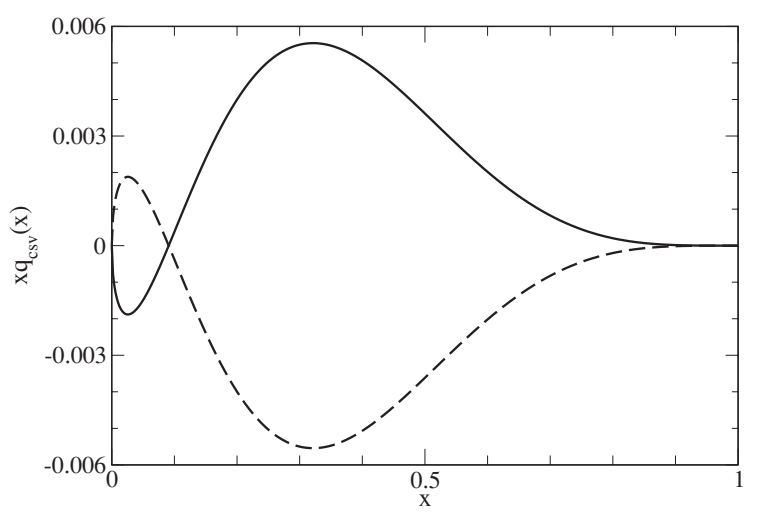

Figure 2. The valence quark CSV function from [3] corresponding to best fit value $\kappa=-0.2$ defined in equation (5). Solid curve: $x \delta d_{\mathrm{v}}(x)$; dashed curve: $x \delta u_{\mathrm{v}}(x)$.

EM corrections is that they can produce a charge symmetry violating contribution to the QCD evolution equations. This has been considered recently by the MRST group [12], and also by Glueck, Jiminez-Delgado and Reya [13]. We will summarize the effects of these 'QED splitting' terms in the following section. A series of EM corrections contribute to producing the $\mathrm{n}-\mathrm{p}$ mass difference $\delta M \equiv M_{\mathrm{n}}-M_{\mathrm{p}}$. Neglecting for the moment QED splitting effects, one can consider the CSV parton distributions as a function of $\delta m$ and $\delta M$. As quark models of the nucleon are explicit functions of the quark and nucleon mass, one can investigate the effect of variations in quark and nucleon masses on valence parton distributions in such models. This technique was used by Sather [14] and Benesh and Londergan [15], and characterizes isospin violation in PDFs through the relation

$$
\delta q_{\mathrm{v}}=\frac{\partial q_{\mathrm{v}}}{\partial m} \delta m+\frac{\partial q_{\mathrm{v}}}{\partial M} \delta M
$$

In equation (7), $\delta m=m_{d}-m_{u} \sim 4 \mathrm{MeV}$ is the down-up quark mass difference, and $\delta M=1.3 \mathrm{MeV}$ is the $\mathrm{n}-\mathrm{p}$ mass difference. Applying this relation to a simple quark model, Sather obtained an analytic approximation relating valence quark CSV and derivatives of the valence PDFs

$$
\begin{aligned}
& \delta d_{\mathrm{v}}(x)=-\frac{\delta M}{M} \frac{\mathrm{d}}{\mathrm{d} x}\left[x d_{\mathrm{v}}(x)\right]-\frac{\delta m}{M} \frac{\mathrm{d}}{\mathrm{d} x} d_{\mathrm{v}}(x) \\
& \delta u_{\mathrm{v}}(x)=\frac{\delta M}{M}\left(-\frac{\mathrm{d}}{\mathrm{d} x}\left[x u_{\mathrm{v}}(x)\right]+\frac{\mathrm{d}}{\mathrm{d} x} u_{\mathrm{v}}(x)\right) .
\end{aligned}
$$

In figure 2 we plot the valence quark CSV distributions obtained by MRST, corresponding to the best fit value $\kappa=-0.2$. The solid curve represents $x \delta d_{\mathrm{v}}(x)$ and the dashed curve is $x \delta u_{\mathrm{v}}(x)$ (which by definition is equal and opposite to the solid curve). In figure 3 we plot the theoretical results of Rodionov et al [16], who carried out a similar theoretical quark model calculation of valence quark CSV but included effects of quark transverse momentum which had been neglected by Sather. The solid curve is $x \delta u_{\mathrm{v}}(x)$ while the dot-dashed curve is $x \delta d_{\mathrm{v}}(x)$, both evolved to $Q^{2}=10 \mathrm{GeV}^{2}$. The sign and relative magnitude of both $\delta d_{\mathrm{v}}(x)$ and $\delta u_{\mathrm{v}}(x)$ are remarkably similar in the theoretical and phenomenological CSV PDFs and the second moments of both distributions are equal to the MRST values to better than $10 \%$ (Sather obtained qualitatively similar results). The excellent agreement with the phenomenological results provides some theoretical support for the functional form chosen by MRST. However, 


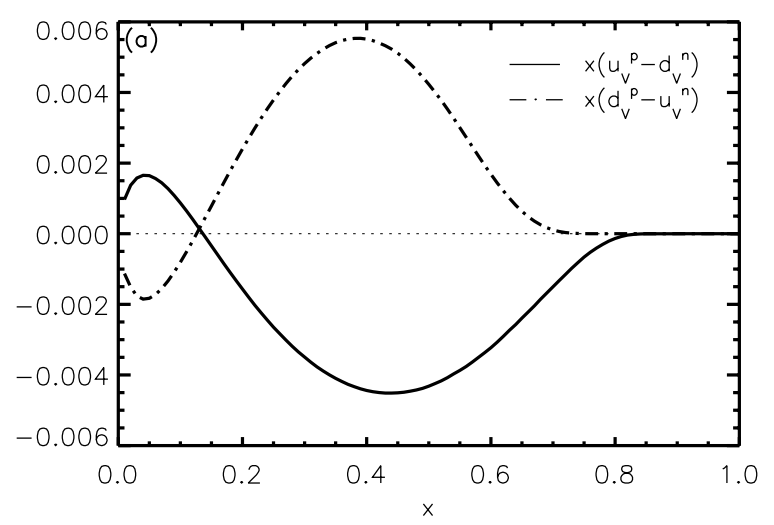

Figure 3. Valence quark CSV contributions, $x \delta q_{\mathrm{v}}(x)$ versus $x$ (solid line: $x \delta u_{\mathrm{v}}$; dash-dot line: $x \delta d_{\mathrm{v}}$ ), calculated by Rodionov et al [16] using MIT bag model wavefunctions evolved to $Q^{2}=$ $10 \mathrm{GeV}^{2}$.

within the $90 \%$ confidence region for the global fit, the valence quark CSV PDFs could be either four times as large as those predicted by Sather and Rodionov, or they could be three times as big with the opposite sign. The great value of the MRST global fit is that CSV distributions with this shape, and with values of $\kappa$ within this range, will not disagree seriously with any of the high energy data used to extract quark and gluon distribution functions.

The MRST group also searched for the presence of charge symmetry violation in the sea quark sector. Again, they chose a specific form for sea quark CSV dependent on a single parameter, namely

$$
\bar{u}^{n}(x)=\bar{d}^{p}(x)[1+\delta] \quad \bar{d}^{n}(x)=\bar{u}^{p}(x)[1-\delta] .
$$

With this form, the net momentum carried by sea quarks in the proton and neutron should be approximately equal; although this statement depends on $Q^{2}$, the momentum carried by sea quarks in the proton and neutron is very similar throughout the kinematic region of interest.

Somewhat surprisingly, evidence for sea quark CSV in the global fit is substantially stronger than that for valence quark CSV. As shown in the top curve in figure 1, the best fit is obtained for $\delta=0.08$, corresponding to an $8 \%$ violation of charge symmetry in the nucleon sea. The $\chi^{2}$ corresponding to this value is substantially better than with no charge symmetry violation, primarily because of the improvement in the fit to the NMC $\mu-D$ DIS data [7, 8] when $\bar{u}^{n}$ is increased. The fit to the E605 Drell-Yan data [17] is also substantially improved by the sea quark CSV term.

We can check the MRST results by comparing them with the charge ratio measurements that were summarized in section 2, where equation (2) relates the CSV parton distributions to the charge ratio $R_{c}$. We have taken the valence and sea quark PDFs from MRST ${ }^{3}$, and in figure 4 they are plotted against the experimental charge ratio obtained using the CCFR charged-current neutrino structure function and the NMC muon structure function. The longdashed curve corresponds to the sea quark CSV term with the best value $\delta=0.08$ from the MRST fit. The remaining curves correspond to various values for valence quark CSV. The solid curve corresponds to the best value $\kappa=-0.2$, the short-dashed curve corresponds to $\kappa=-0.8$ and the dash-dot curve corresponds to $\kappa=+0.65$. The latter two correspond to

3 When MRST [3] carry out the global fits allowing either valence or sea CSV, the best-fit PDFs change slightly from the values obtained when charge symmetry is enforced. In our calculations we have used the modified PDFs supplied to us by R S Thorne [45]. 


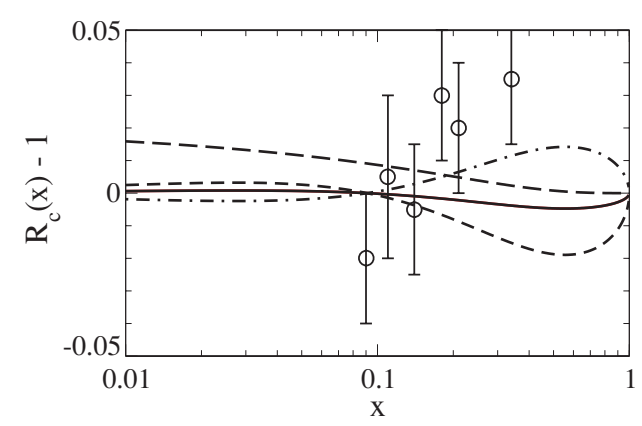

Figure 4. The 'charge ratio' $R_{c}-1$ defined in equation (2), compared with charge symmetry violating PDFs obtained by the MRST global fit [3]. Data are obtained from CCFR neutrino cross sections of [6] and NMC muon DIS, [7, 8]. Long-dashed curve: sea quark CSV with best fit parameter $\delta=0.08$ in equation (9); solid curve: valence quark CSV corresponding to best fit value $\kappa=-0.2$ in equation (5); short dashed curve: valence quark CSV for $\kappa=-0.8$; dash-dot curve: valence quark CSV for $\kappa=+0.65$.

values of $\kappa$ at the $90 \%$ confidence level for the MRST fit to valence quark CSV. For values $x<0.085$, we have not included the charge ratio experimental data since these changed significantly in the CCFR re-analysis [9], and the low- $x$ data are subject to large NLO effects.

Both the valence and sea quark CSV distributions of MRST are in good agreement with the experimental limits from the CCFR/NMC data. At every $x$ value the phenomenological values are within two standard deviations of the data. This is understandable, since both the NMC and CCFR data were included in the MRST global fit that extracted the CSV parameters. We have not included overall normalization errors, of roughly $2 \%$, on both the CCFR and NMC data. Since the best fit valence quark PDFs of MRST (the solid curve in figure 4) are very close to the theoretical predictions of Sather and Rodionov, the theoretical predictions are well within the upper limits on parton CSV from the best experiments. CSV effects in reasonable agreement with high energy data are substantially larger than predicted by theory; valence CSV effects could be four times as large as predicted by Sather or Rodionov (or three times as large with the opposite sign), and sea quark CSV effects are also significantly larger than theoretical predictions [15].

The only remaining issue is whether the theoretical and phenomenological PDFs agree with the limits on parton momentum estimated by MRST as relative errors of about $2 \%$. The best value obtained by MRST was $\kappa=-0.2$. With this value, one obtains the relative momentum change for valence neutrons

$$
\frac{\left|\delta U_{\mathrm{v}}\right|}{U_{\mathrm{v}}+D_{\mathrm{v}}}=\frac{\delta D_{\mathrm{v}}}{U_{\mathrm{v}}+D_{\mathrm{v}}} \approx 0.5 \%
$$

This value is well within experimental limits. Even using the largest value of $\kappa$ within the $90 \%$ confidence limit, $\kappa=-0.8$, leads to a momentum change of only $2 \%$. Thus all of the phenomenological valence CSV distributions (and the theoretical distributions of Sather and Rodionov et al) correspond to momentum values within experimental limits.

\section{Charge symmetry violation and neutrino DIS reactions}

The NuTeV experimental group [4] extracted a value for the Weinberg angle $\sin ^{2} \theta_{W}$ using a procedure originally suggested by Paschos and Wolfenstein [18]. By measuring total 
neutral-current (NC) and charge-changing (CC) neutrino cross sections on isoscalar targets one obtains

$$
R^{-} \equiv \frac{\left\langle\sigma_{N C}^{\nu N_{0}}\right\rangle-\left\langle\sigma_{N C}^{\bar{\nu} N_{0}}\right\rangle}{\rho_{0}^{2}\left(\left\langle\sigma_{C C}^{\nu N_{0}}\right\rangle-\left\langle\sigma_{C C}^{\bar{\nu} N_{0}}\right\rangle\right)}=\frac{1}{2}-\sin ^{2} \theta_{W}
$$

In equation (11), $\left\langle\sigma_{N C}^{\nu N_{0}}\right\rangle$ and $\left\langle\sigma_{C C}^{\nu N_{0}}\right\rangle$ are respectively the $\mathrm{NC}$ and $\mathrm{CC}$ inclusive, total cross sections for neutrinos on an isoscalar target. The quantity $\rho_{0} \equiv M_{W} /\left(M_{Z} \cos \theta_{W}\right)$ is one in the standard model.

From measurements of $v$ and $\bar{v} \mathrm{CC}$ and NC cross sections on iron [4], NuTeV extracted a value $\sin ^{2} \theta_{W}=0.2277 \pm 0.0013$ (stat) \pm 0.0009 (syst), a result three standard deviations above the measured fit to other electroweak processes, $\sin ^{2} \theta_{W}=0.2227 \pm 0.00037$ [19]. It has proved quite difficult to account for the $\mathrm{NuTeV}$ result through 'new physics' beyond the standard model [20], so recent investigations have focused on 'QCD corrections' to the NuTeV result, including CSV corrections [21].

The NuTeV group provides functionals that allow one to calculate the magnitude of various QCD corrections [22]. Using the NuTeV functionals, the CSV correction to the Weinberg angle was found to be $\Delta R_{C S V}=-0.0017$ and -0.0015 using the Sather [14] and Rodionov [16] theoretical PDFs, and $\Delta R_{C S V}=-0.0017$ using the phenomenological PDFs of MRST [3] with the best value $\kappa=-0.2$. There is close agreement between the theoretical predictions and phenomenological results. The CSV corrections tend to remove roughly one-third of the discrepancy between the NuTeV result and the Weinberg angle extracted from the extremely precise data obtained from electron-positron colliders near the $Z$ mass. Recently, the MRST group [12] and Glueck, Jiminez-Delgado and Reya [13] have considered the explicit coupling of photons to quarks in the QCD evolution equations. One includes terms where a quark radiates a photon, in analogy to the familiar case where a quark radiates a gluon. This adds a photon distribution into the DGLAP evolution equations [23-25]. Since the photon couples differently to up and down quarks, this produces an additional source of CSV in parton distributions. The effect occurs even if the PDFs are charge symmetric at the starting value $Q_{0}^{2}$ and introduces an explicit $Q^{2}$ dependence into the CSV effects. The resulting CSV effect should be added to the theoretical CSV contributions calculated from quark models. Although the MRST and Glueck calculations are somewhat different, their CSV contributions, evolved to the $Q^{2}$ appropriate for the NuTeV experiment, agree rather well. Both groups find a contribution from QED splitting that would remove about one-third of the NuTeV anomaly. Together, the theoretical quark model and QED splitting CSV effects remove roughly two-thirds of the NuTeV anomaly.

The large uncertainty in phenomenological valence quark CSV found by MRST means that, within the limits of the MRST global fit, the isospin violating contribution to the Weinberg angle could be quite substantial. The value $\kappa=-0.6$ within the MRST $90 \%$ confidence limit, would remove all of the Weinberg angle anomaly, while the value $\kappa=+0.6$ would double the discrepancy.

For the most part, other QCD corrections to the NuTeV Weinberg angle measurement are small and/or well understood. The correction for the neutron excess in iron is large, but apparently well known [20, 22]. Radiative corrections are also substantial [26]; they have been re-analysed, but the new corrections have not yet been applied to the NuTeV analysis [27]. Leading twist parton shadowing and antishadowing effects could remove roughly $20 \%$ of the Weinberg angle anomaly [28], while higher twist contributions associated with vector meson dominance could be significant [29]. The situation with respect to strange quarks is uncertain. Spontaneously broken chiral $\mathrm{SU}(3) \times \mathrm{SU}(3)$ symmetry implies an asymmetry 
between $s(x)$ and $\bar{s}(x)[30,31]$. This asymmetry produces a correction to the Weinberg angle that is proportional to the strange momentum asymmetry

$$
S_{\mathrm{v}}=\int_{0}^{1} x[s(x)-\bar{s}(x)] \mathrm{d} x .
$$

A positive (negative) value for $S_{\mathrm{v}}$, meaning that strange quarks carry more (less) net momentum than strange antiquarks, would decrease (increase) the size of the Weinberg anomaly. The $s$ and $\bar{s}$ distributions can be extracted from measurements of opposite sign dimuons in $v$ or $\bar{v}$ reactions measured by the CCFR [32] and NuTeV [33] collaborations. From an analysis of these reactions, NuTeV obtain values for $S_{\mathrm{v}}$ that are consistent with zero, or perhaps small and negative [22]. However, the CTEQ group [34-36] has included the dimuon data in a global fit of PDFs; they obtain positive values for $S_{\mathrm{v}}$ that could account for roughly one-third of the Weinberg angle anomaly. The NuTeV and CTEQ groups are currently collaborating on analysing the data, although at present the qualitative differences persist. Catani et al [37] have shown that a strange asymmetry can be generated perturbatively at three-loop order. The size of this effect should be a small fraction of the NuTeV anomaly, and it will increase the anomaly.

\section{Charge symmetry violating contributions to sum rules}

Sum rules can provide extremely useful information on parton distributions. By taking the integral of quark structure functions over $x$, we can invoke the quark normalization conditions on both the valence quark distributions and on valence quark CSV. Consequently, terms that contribute to lowest-order sum rules will depend on integer valence quark normalizations, plus contributions from the first moment of antiquark distributions and sea quark CSV [5]. We first review the Adler and Gottfried sum rules including the role of parton charge symmetry. Then we use the MRST isospin violating PDFs to estimate possible contributions to these sum rules.

\subsection{Charge symmetry violating contribution to the Gottfried sum rule}

The Gottfried sum rule $S_{G}$ [38] is obtained by comparing the $F_{2}$ structure functions for charged-leptons on protons and neutrons. It is defined as

$$
\begin{aligned}
S_{G} & \equiv \int_{0}^{1} \frac{\mathrm{d} x}{x}\left[F_{2}^{\mu p}\left(x, Q^{2}\right)-F_{2}^{\mu n}\left(x, Q^{2}\right)\right] \\
& =\frac{1}{3}-\frac{2}{3}\left\langle\bar{d}^{p}-\bar{u}^{p}\right\rangle+\frac{8}{9}\langle\delta \bar{d}\rangle+\frac{2}{9}\langle\delta \bar{u}\rangle .
\end{aligned}
$$

In equation (13), we have used valence quark normalization and the assumption that $\left\langle s^{p}-s^{n}\right\rangle=0$. The Gottfried sum rule was measured experimentally by the NMC group $[7,8]$, who extracted the $F_{2}$ structure functions for muon DIS on proton and deuteron targets and obtained $S_{G}=0.235 \pm 0.026$.

The experimental Gottfried sum rule is four standard deviations below the naive value $1 / 3$. From equation (13), this implies contributions from flavour symmetry violation in the proton sea, $\bar{d}^{p} \neq \bar{u}^{p}$ [39], and/or isospin violation in sea quark PDFs. Direct measurement of the $\bar{d}-\bar{u}$ asymmetry in the proton was obtained first from the NA51 comparison of DrellYan $p p$ and $p D$ at a single $x$ value [40]. This was then definitively measured by the E866 Collaboration who used Drell-Yan $p p$ and $p D$ data to map out $\bar{d}-\bar{u}$ versus $x$ [41, 42]. The E866 group obtained

$$
\langle\bar{d}-\bar{u}\rangle \equiv\langle\Delta\rangle=0.118 \pm 0.012 .
$$


Subsequently, the HERMES group confirmed the E866 results through measurements of electron-induced semi-inclusive DIS [43].

We first review results obtained for the Gottfried sum rule with the MRST2001 [44] global fit PDFs with no isospin violation. In this parametrization, the sea quark distributions at the starting scale $Q_{0}^{2}=1 \mathrm{GeV}^{2}$ are given by

$$
\begin{aligned}
& \bar{d}^{p}(x)=0.2 S(x)+\frac{\Delta(x)}{2}, \quad \bar{u}^{p}(x)=0.2 S(x)-\frac{\Delta(x)}{2} \\
& x S(x)=0.222 x^{-0.26}(1-x)^{7.10}\left(1+3.42 x^{0.5}+10.30 x\right) \\
& x \Delta(x)=1.195 x^{1.24}(1-x)^{9.10}\left(1+14.05 x-45.52 x^{2}\right) .
\end{aligned}
$$

With this parametrization for the parton PDFs, MRST obtains

$$
S_{G}=1 / 3-2 / 3\langle\Delta\rangle=0.266 .
$$

The MRST result for the Gottfried sum rule is just over $1 \sigma$ above the NMC value. The MRST value for the first moment of the $\bar{d}-\bar{u}$ asymmetry in the proton is consistent with the experimental result obtained from the E866 Drell-Yan $p p$ and $p D$ data.

In principle, one could insert the sea quark CSV distributions obtained by MRST, and calculate the contribution from sea quark CSV to the Gottfried sum rule. However, if we use the functional form of sea quark CSV assumed by MRST, equation (9), we obtain an infinite result for $S_{G}$. This is due to the fact that the sea quark CSV is assumed to be proportional to the sea quark PDFs, which have infinite first moment. Although the Gottfried sum rule is not required to be finite, it is commonly expected that the $F_{2}$ structure functions for the neutron and proton should agree at small $x$, based on arguments from Regge theory. The MRST group plans to carry out future global fits assuming a modified functional form for sea quark CSV [45]. The modified functional form would produce finite CSV contributions to the Gottfried sum rule.

\subsection{Charge symmetry violation and the Adler sum rule}

The Adler sum rule [46] is given by the integral of the $F_{2}$ structure functions for charged-current $v$ and $\bar{v}$ scattering on a proton target. The Adler sum rule, $S_{A}$, is defined as

$$
\begin{aligned}
S_{A} & \equiv \lim _{Q^{2} \rightarrow \infty} \int_{0}^{1} \mathrm{~d} x\left[\frac{F_{2}^{W^{-} p}\left(x, Q^{2}\right)-F_{2}^{W^{+} p}\left(x, Q^{2}\right)}{2 x}\right] \\
& =\int_{0}^{1} \mathrm{~d} x\left[u_{\mathrm{v}}^{p}(x)-d_{\mathrm{v}}^{p}(x)\left(1-\left|V_{t d}\right|^{2}\right)-(s(x)-\bar{s}(x))\right]=1 .
\end{aligned}
$$

Since the proton has zero net strangeness, the Adler sum rule should give $S_{A}=1$ - up to the very small term $\left|V_{t d}\right|^{2} \approx 1 \times 10^{-4}$. The Adler sum rule involves dividing the $F_{2}$ structure functions by $x$, which emphasizes the contribution from small $x$. The Adler sum rule then follows from the normalization of the valence quark distributions. As a consequence of the algebra of SU(2) charges, the Adler sum rule has no QCD corrections.

The best experimental results for the Adler sum rule come from the WA25 experiment [47] which used the BEBC $\mathrm{H}$ and D bubble chambers at CERN and obtained data for values of $Q^{2}$ between 1 and $20 \mathrm{GeV}^{2}$. Within rather large experimental errors, the results are independent of $Q^{2}$ with an average value $S_{A}=1.01 \pm 0.08 \pm 0.18$. However, these results need to be renormalized [48] to bring the total $v N$ cross section used by the WA25 group into agreement with currently accepted values $[49,50]$. Following renormalization of the WA25 cross sections, the result becomes $S_{A}=1.08 \pm 0.08 \pm 0.18$. 
The large errors arise from the factor $1 / x$ in the sum rule, equation (17), which gives a strong weighting to small- $x$ data. The bubble chamber data in this region are scarce and the error bars are relatively large. The prospect for future 'neutrino factories' with very high rates raises the possibility of much more precise tests of the Adler sum rule.

Since the Adler sum rule defined in equation (17) requires $v$ and $\bar{v}$ reactions on protons, it has no contribution from CSV effects. However, experiments typically obtain many more events from neutrinos than antineutrinos, hence the existing experiments compare $v$ cross sections on neutrons (e.g., deuterons) and protons. This follows from the relation

$$
F_{2}^{W^{+} n}\left(x, Q^{2}\right)=F_{2}^{W^{-} p}\left(x, Q^{2}\right)+2 x[s(x)-\bar{s}(x)-\delta u(x)-\delta \bar{d}(x)],
$$

which is true in lowest order. Thus, the WA25 measurement does not construct the Adler sum rule $S_{A}$ of equation (17), but instead measures a different quantity

$$
\begin{aligned}
\widetilde{S}_{A} & \equiv \int_{0}^{1} \mathrm{~d} x\left[\frac{F_{2}^{W^{+} n}\left(x, Q^{2}\right)-F_{2}^{W^{+} p}\left(x, Q^{2}\right)}{2 x}\right] \\
& =\int_{0}^{1} \mathrm{~d} x\left[u_{\mathrm{v}}^{p}(x)-d_{\mathrm{v}}^{p}(x)-\delta u(x)-\delta \bar{d}(x)\right] \\
& =1-\int_{0}^{1} \mathrm{~d} x[\delta \bar{u}(x)+\delta \bar{d}(x)] .
\end{aligned}
$$

The sum rule $\widetilde{S}_{A}$ is equal to the Adler sum rule of equation (17) if parton charge symmetry holds. Thus the 'Adler sum rule' measured by the WA25 group should equal one only if the first moment of this combination of sea quark CSV terms vanishes, i.e.

$$
\langle\delta \bar{u}+\delta \bar{d}\rangle=0 \text {. }
$$

Conversely, assuming that the Adler sum rule should be one, then the experimental results allow us to place an upper limit on the first moment of the sea quark CSV amplitudes in equation (20) at the $20 \%$ level. We can use the phenomenological sea quark CSV amplitudes determined by MRST to estimate the CSV contribution to the WA25 measurement. Inserting the MRST sea quark function of equation (9) into the Adler sum rule, we obtain

$$
\begin{aligned}
\widetilde{S}_{A} & =1-\int_{0}^{1} \mathrm{~d} x[\delta \bar{u}(x)+\delta \bar{d}(x)] \\
& =1+\delta\left\langle\bar{d}^{p}-\bar{u}^{p}\right\rangle=1.0081 .
\end{aligned}
$$

With the MRST ansatz for sea quark CSV, there would be a difference of less than $1 \%$ between the Adler sum rule and the quantity measured in the WA25 experiment. Note that the contribution from sea quark CSV is highly sensitive to the functional form assumed by MRST.

\section{Conclusions}

In conclusion, we have reviewed the charge symmetry violating PDFs obtained by the MRST group through a global fit to high energy data. We have compared this to the most stringent experimental test of parton charge symmetry, the 'charge ratio' comparison of the $F_{2}$ structure functions for charged-lepton DIS and for neutrino charged-current DIS, in principle on isoscalar targets. The magnitude of charge symmetry violation obtained by MRST is within the limits provided by the charge ratio test-not surprisingly, since the data sets for both the charged-lepton DIS and neutrino charged-current reactions were included in the global fit used by MRST. We also compared the valence quark CSV PDFs obtained by MRST with 
theoretical predictions. While the best fit MRST value agrees strikingly well with theory, the limits allowed by the MRST fit are substantially larger than theory and could well have the opposite sign to the theoretical PDFs.

We then reviewed the contribution of charge symmetry violating PDFs to the value of the Weinberg angle extracted from the NuTeV experiment [4]. The magnitude of CSV effects allowed by the MRST global fit makes charge symmetry violation perhaps the most likely single effect that could explain the anomalous value for the Weinberg angle found by NuTeV. CSV effects will also contribute to measurements of the Gottfried sum rule. We showed that current experiments do not measure the Adler sum rule, but a different quantity that equals the Adler sum rule under the assumption of parton charge symmetry. Both the Gottfried and 'Adler' sum rules are sensitive to different linear combinations of the first moment of sea quark CSV terms.

If parton CSV effects are sufficiently large to remove the Weinberg angle anomaly, such effects should be visible in various other experiments. We had previously suggested several experiments that could potentially reveal the presence of charge symmetry violation in parton distributions [5]. However, the estimates of those effects were based on theoretical calculations that predicted substantially smaller CSV effects than those allowed by MRST. In addition, while theoretical models are likely to give reasonable predictions for valence quark CSV, it is substantially more difficult to obtain reliable estimates of sea quark CSV. In the MRST global fits allowing isospin violation in PDFs, the evidence for sea quark CSV was significantly stronger than for valence quark CSV. It is clearly of great interest now to investigate this issue experimentally. The possibility of a future neutrino factory would be particularly promising for future high-precision tests of the Adler sum rule.

\section{Acknowledgments}

This work was supported in part (JTL) by National Science Foundation research contract PHY0302248 and by (AWT) DOE contract DE-AC05-84ER40150, under which SURA operates Jefferson Laboratory. The authors wish to thank G P Zeller and K McFarland of the NuTeV collaboration, W Melnitchouk at JLab and R S Thorne from the MRST group, for useful discussions regarding the issues presented here.

\section{References}

[1] Miller G A, Nefkens B M K and Slaus I 1990 Phys. Rep. 1941

[2] Henley E M and Miller G A 1979 Mesons in Nuclei ed M Rho and D H Wilkinson (Amsterdam: North-Holland)

[3] Martin A D, Roberts R G, Stirling W J and Thorne R S 2004 Eur. Phys. J. C 35325

[4] Zeller G P et al 2002 Phys. Rev. Lett. 88091802

[5] Londergan J T and Thomas A W 1998 Prog. Part. Nucl. Phys. 4149

[6] Seligman W G et al (CCFR Collaboration) 1997 Phys. Rev. Lett. 791213

[7] Amaudruz P et al (NMC Collaboration) 1991 Phys. Rev. Lett. 662712 Amaudruz P et al (NMC Collaboration) 1992 Phys. Lett. B 295159

[8] Arneodo M et al (NMC Collaboration) 1997 Nucl. Phys. B 4833

[9] Yang U K et al (CCFR Collaboration) 2001 Phys. Rev. Lett. 862742

[10] Georgi H and Politzer H D 1976 Phys. Rev. D 141829 Barnett R M 1976 Physica D 1470

[11] Boros C, Steffens F M, Londergan J T and Thomas A W 1999 Phys. Lett. B 468161

[12] Martin A D, Roberts R G, Stirling W J and Thorne R S 2005 Eur. Phys. J. C 39155

[13] Glueck M, Jiminez-Delgado P and Reya E 2005 Phys. Rev. Lett. 95022002 (Preprint hep-ph/0501169) Glueck M, Jiminez-Delgado P and Reya E 2005 Preprint hep-ph/0503103

[14] Sather E 1992 Phys. Lett. B 274433 
[15] Benesh C J and Londergan J T 1998 Phys. Rev. C 581218

[16] Rodionov E N, Thomas A W and Londergan J T 1994 Mod. Phys. Lett. A 91799

[17] Moreno G et al (E605 Collaboration) 1991 Phys. Rev. D 432815

[18] Paschos E A and Wolfenstein L 1973 Phys. Rev. D 791

[19] Abbaneo D et al 2001 CERN Report CERN-EP/2001-098 Preprint hep-ex/0112021

[20] Davidson S, Forte S, Gambino P, Rius N and Strumia A 2002 J. High Energy Phys. JHEP02(2002)037

[21] Londergan J T 2005 Nucl. Phys. (Proc. Suppl.) B 14168

[22] Zeller G P et al 2002 Phys. Rev. D 65111103

[23] Gribov V N and Lipatov L N 1972 Sov. J. Nucl. Phys. 15438

[24] Dokshitzer Yu L 1977 Sov. Phys.-JETP 46641

[25] Altarelli G and Parisi G 1977 Nucl. Phys. B 126298

[26] Bardin D Yu and Dokuchaeva V A, Report JINR-E2-86-260

[27] Diener K-P O, Dittmaier S and Hollik W 2004 Phys. Rev. D 69073005

[28] Brodsky S J, Schmidt I and Yang J-J 2004 Phys. Rev. D 70116003

[29] Miller G A and Thomas A W 2005 Int. J. Mod. Phys. A 2095

[30] Signal A I and Thomas A W 1987 Phys. Lett. B 191205

[31] Thomas A W, Melnitchouk W and Steffens F M 2000 Phys. Rev. Lett. 852892

[32] CCFR Collaboration, Bazarko A O et al 1995 Z. Phys. C 65189

[33] Goncharov M et al (NuTeV Collaboration) 2001 Phys. Rev. D 64112006

[34] Kretzer S 2004 Talk presented at XXXIX Rencontres de Montreal Preprint hep-ph/0405221

[35] Olness F, Pumplin J, Stump D, Huston J, Nadolsky P, Lai H L, Kretzer S, Owens J F and Tung W K 2005 Eur. Phys. J. C 40145 (Preprint hep-ph/0312323)

[36] Kretzer S, Olness F, Pumplin J, Reno M H, Stump D and Tung W K 2004 Phys. Rev. Lett. 93041802

[37] Catani S, de Florian D, Rodrigo G and Vogelsang W 2004 Phys. Rev. Lett. 93152003

[38] Gottfried K 1967 Phys. Rev. Lett. 181174

[39] Thomas A W 1983 Phys. Lett. B 12697

[40] Baldit A et al (NA51 Collaboration) 1994 Phys. Lett. B 332244

[41] Hawker E A et al (E866 Collaboration) 1998 Phys. Rev. Lett. 803715

[42] Towell R S et al (E866 Collaboration) 2001 Phys. Rev. D 64052002

[43] Ackerstaff K et al (HERMES Collaboration) 1998 Phys. Rev. Lett. 815519

[44] Martin A D, Roberts R G, Stirling W J and Thorne R S 2002 Eur. Phys. J. C 2373

[45] Thorne R S, Private communication

[46] Adler S L 1966 Phys. Rev. 1431144

[47] Allasia D et al (WA25 Collaboration) 1984 Phys. Lett. B 135231

Allasia D et al 1985 Z. Phys. C 28321

[48] Sterman Get al 1995 Rev. Mod. Phys. 67157

[49] Blair R et al 1983 Phys. Rev. Lett. 51343

[50] Berge P et al 1987 Z. Phys. C 35443 